\title{
Tau method for the numerical solution of a fuzzy fractional kinetic model and its application to the oil palm frond as a promising source of xylose
}

\begin{abstract}
The Oil Palm Frond (a lignocellulosic material) is a high-yielding energy crop that can be utilized as a promising source of xylose. It holds the potential as a feedstock for bioethanol production due to being free and inexpensive in terms of collection, storage and cropping practices. The aim of the paper is to calculate the concentration and yield of xylose from the acid hydrolysis of the Oil Palm Frond through a fuzzy fractional kinetic model. The approximate solution of the derived fuzzy fractional model is achieved by using a tau method based on the fuzzy operational matrix of the generalized Laguerre polynomials. The results validate the effectiveness and applicability of the proposed solution method for solving this type of fuzzy kinetic model.
\end{abstract}

Keyword: Fuzzy differential equations; Caputo fractional derivatives; Spectral tau method; Laguerre polynomials; Operational matrices 